\title{
In-plane dipole coupling anisotropy of a square ferromagnetic Heisenberg monolayer
}

\author{
M. Dantziger, B. Glinsmann, S. Scheffler, B. Zimmermann, and P. J. Jensen \\ I. Institut für Theoretische Physik, Universität Hamburg, \\ Jungiusstr.9, D-20355 Hamburg, Germany
}

\begin{abstract}
In this study we calculate the dipole-coupling-induced quartic in-plane anisotropy of a square ferromagnetic Heisenberg monolayer. This anisotropy increases with an increasing temperature, reaching its maximum value close to the Curie temperature of the system. At $T=0$ the system is isotropic, besides a small remaining anisotropy due to the zero-point motion of quantum mechanical spins. The reason for the dipole-coupling-induced anisotropy is the disturbance of the square spin lattice due to thermal fluctuations ('order-by-disorder' effect). For usual ferromagnets its strength is small as compared to other anisotropic contributions, and decreases by application of an external magnetic field. The results are obtained from a Heisenberg Hamiltonian by application of a mean field approach for a spin cluster, as well as from a many-body Green's function theory within the Tyablikov-decoupling (RPA).

PACS numbers: 75.70.-i, 75.50.Ee, 75.70.Cn
\end{abstract}

\section{INTRODUCTION}

The investigation of the magnetic properties of ferromagnetic ultrathin films is a field of intense current interestl. In this contribution we determine the magnetic in-plane anisotropy of a square two-dimensional (2D) Heisenberg ferromagnet in presence of the magnetic dipole interaction. In 2D magnets the action of magnetic anisotropies is trofold. First, they induce a long range magnetization $\mathrm{B}^{-1}$ with a Curie temperature of the order of the exchange coupling $\bar{E}$, whereas a strictly isotropic 2D Heisenberg magnet does not exhibit any long range magneticorder at finite temperatures (Mermin-Wagnertheorem)6. Moreover, the long range character of the dipole interaction itself is sufficient to stabilize the magnetization in a 2D magnet. Secondly, the anisotropies determine the easy and hard axes of the magnetization with respect to the lattice frame 1 . For a ferromagnetic thin film the dipole coupling prefers an in-plane direction of the magnetization (shape anisotropy, demagnetizing field). Except for very small thicknesses thin films are usually in-plane magnetized. The corresponding in-plane easy axis depends on the lattice symmetry of the film face. For a rectangular (110) monolayer the dipole interaction induces a uniaxial in-plane anisotropy, the easy axis being the in-plane axial direction with the smaller lattice constant, as can easily be shown by calculating the corresponding lattice sums.

On the other hand, for a square monolayer the dipole coupling, although itself not rotational invariant, exhibits a continuous energy degeneracy for classical spins at $T=0$. Thus, due to the Mermin-Wagner theorem a long range magnetic order is not expected at finite temperatures. However, as has been shown by Monte Carlo calculations and interacting spin wave theory, a magnetic ordering and a critical temperature exists for dipole coupled spins on a square latticed, since the magnetic excitations are not continuously degenerate. In this case a quartic in-plane anisotropy is present, the corresponding easy axes being the edges of the square lattice. In other words, the density of states and thus the entropy depends on the magnetic direction within the lattice. This phenomenon is an example of the 'order-by-disorder' effect in frustrated magnets 8 . In these systems thermal fluctuations or lattice disturbances partly remove frustrations, and a collective magnetic ordering emerges at finite temperatures.

Whereas the existence of a finite magnetization and a quartic anisotropy at finite temperatures for dipole coupled spins on a square lattice has been proven, the strength and the temperature behavior of this anisotropy has not been determined yet. Of particular interest is whether the dipole-coupling-induced quartic anisotropy can be measured, and how this anisotropy compares with the single-ion quartic lattice anisotropy due to the spinorbit coupling. In this study we will adress these questions with the help of a mean field approximation, taking into account a square spin cluster with different numbers of spins (Oguchi-theory) 10 , as outlined in Sec.II. Furthermore, we apply a many-body Green's function theory within the Tyablikov-decoupling (RPA) by considering collective magneticercitations (spin waves) and interactions between them 11.12 , which are in particular important for 2D magnetic systems. Our results are summarized and discussed in Sec.III.

\section{THEORY}

The free energy $F(T, \phi)$ is determined within a Heisenberg-type Hamiltonian as a function of the temperature $T$ and the in-plane angle $\phi$. A square (001) ferromagnetic monolayer is considered, which is spanned by the $x z$-plane, the $z$-direction refers to $\phi=0$. The isotropic nearest neighbor Heisenberg exchange coupling $J$, the magnetic dipole-dipole interaction, and an external magnetic field $\mathbf{B}$ between localized Heisenberg spins 
$\mathbf{S}_{i}$ on lattice sites $i$ are taken into account:

$$
\begin{gathered}
\mathcal{H}=-\frac{J}{2} \sum_{\langle i, j\rangle} \mathbf{S}_{i} \mathbf{S}_{j}-g \mu_{B} \sum_{i} \mathbf{B} \mathbf{S}_{i} \\
+\frac{\left(g \mu_{B}\right)^{2}}{2} \sum_{\substack{i, j \\
i \neq j}} \frac{1}{r_{i j}^{5}}\left[r_{i j}^{2} \mathbf{S}_{i} \mathbf{S}_{j}-3\left(\mathbf{r}_{i j} \mathbf{S}_{i}\right)\left(\mathbf{r}_{i j} \mathbf{S}_{j}\right)\right],
\end{gathered}
$$

with $g$ the Landé factor and $\mu_{B}$ the Bohr magneton. The spin quantum number is assumed to be $S=1 / 2$. The lattice vector between sites $i$ and $j$ is given by $\mathbf{r}_{i j}$, with $r_{i j}=\left|\mathbf{r}_{i j}\right|$ the distance. All spins are assumed to be aligned parallely (ferromagnetic order), the magnetization $\mathbf{m}_{i}(T)=\mathbf{m}(T)=(0,0, m(T))$ is directed along the magnetic field $\mathbf{B}=(0,0, B)$.

To account for a varying angle of the magnetization with respect to the lattice frame, the lattice is rotated by the in-plane angle $\phi$ with respect to the $z$ - axis, yielding the rotated lattice vectors:

$$
\mathbf{r}_{i j}^{\prime}=\left(\begin{array}{c}
r_{i j}^{z} \sin \phi+r_{i j}^{x} \cos \phi \\
0 \\
r_{i j}^{z} \cos \phi-r_{i j}^{x} \sin \phi
\end{array}\right) .
$$

Clearly, the only source in Eq.(11) for a possible in-plane anisotropy is the double scalar product of the dipole interaction. Within the single-spin (Bragg-Williams-) mean field approximation 10 the dipole coupling yields an additional contribution to the molecular field. The resulting single-spin Hamiltonian reads

$$
\begin{aligned}
\mathcal{H}^{(1)}= & -\left(q J m(T)+w S(0,0) m(T)+g \mu_{B} B\right) S_{1}^{z} \\
& +\frac{1}{2} m^{2}(T)(q J+w S(0,0))
\end{aligned}
$$

with $q=4$ the number of nearest neighbors (coordination number $), w=\left(g \mu_{B}\right)^{2} / a_{0}^{3}$ the strength of the dipole interaction, $a_{o}$ the lattice constant, $S(0,0) \sim 4.51681$ the corresponding lattice sum 13 , and $m(T)=\left\langle S_{1}^{z}\right\rangle$ the magnetization. Thus, within this approximation the dipole interaction is isotropic for a square monolayer and does not depend on the in-plane angle $\phi$.

Our approach will now be improved by applying the so-called Oguchi methodste. A number $N>1$ of neighboring spins is considered, the interactions between the $N$ spins in this cluster are treated exactly. The remaining lattice is coupled to the spin cluster by a molecular field. To conserve the symmetry of the square lattice, we take into account only square-shaped spin clusters, the three smallest possible clusters are characterized by $N=4,9$, and 16 . Note that the resulting cluster Hamiltonians $\mathcal{H}^{(N)}$ are usually non-diagonal, i.e. contributions proportional to the $S^{x}$ - and $S^{y}$ - spin components may be present. Therefore, as a further approximation we consider only the diagonal elements. This approach guarantees that the magnetization is directed along the external magnetic field. As an example, the Hamiltonian for the four-spin cluster $(N=4)$ reads:

$$
\begin{aligned}
& \mathcal{H}^{(4)}=-(J-w)\left(S_{1}^{z} S_{2}^{z}+S_{1}^{z} S_{3}^{z}+S_{2}^{z} S_{4}^{z}+S_{3}^{z} S_{4}^{z}\right)-\frac{w}{2^{5 / 2}}\left(S_{1}^{z} S_{4}^{z}+S_{2}^{z} S_{3}^{z}\right) \\
&-3 w\left[\sin ^{2} \phi\left(S_{1}^{z} S_{2}^{z}+S_{3}^{z} S_{4}^{z}\right)+\cos ^{2} \phi\left(S_{1}^{z} S_{3}^{z}+S_{2}^{z} S_{4}^{z}\right)+\frac{2 \cos \phi \sin \phi}{2^{5 / 2}}\left(S_{1}^{z} S_{4}^{z}-S_{2}^{z} S_{3}^{z}\right)\right] \\
&-\left(2 J m(T)+g \mu_{B} B\right)\left(S_{1}^{z}+S_{2}^{z}+S_{3}^{z}+S_{4}^{z}\right)-w m(T)\left(S_{1}^{z}+S_{4}^{z}\right)\left(S(0,0)-1-\frac{1}{2^{5 / 2}}(1+6 \cos \phi \sin \phi)\right) \\
&-w m(T)\left(S_{2}^{z}+S_{3}^{z}\right)\left(S(0,0)-1-\frac{1}{2^{5 / 2}}(1-6 \cos \phi \sin \phi)\right)+4 J m^{2}(T)+2 w m^{2}(T)\left(S(0,0)-1-\frac{1}{2^{5 / 2}}\right) .
\end{aligned}
$$

The partition function for this case is given by:

$$
Z^{(4)}(T, B, \phi)=\sum_{S_{1}^{z}, S_{2}^{z}, S_{3}^{z}, S_{4}^{z}=-S}^{+S} \exp \left(-\beta \mathcal{H}^{(4)}\right),
$$

and the (average) magnetization by:

$$
\begin{gathered}
m(T, B, \phi)=\frac{1}{4 Z^{(4)}(T, B, \phi)} \times \\
\sum_{S_{1}^{z}, S_{2}^{z}, S_{3}^{z}, S_{4}^{z}=-S}^{+S}\left(S_{1}^{z}+S_{2}^{z}+S_{3}^{z}+S_{4}^{z}\right) \exp \left(-\beta \mathcal{H}^{(4)}\right),
\end{gathered}
$$

with $\beta=1 / k_{B} T$ and $k_{B}$ the Boltzmann constant. The corresponding expressions for the other investigated sizes are rather lengthy and not shown here. The difference of the free energy $F(T, B, \phi)=-k_{B} T \ln Z(T, B, \phi)$ between the diagonal $(\phi=\pi / 4)$ and the axial directions $(\phi=0)$ yields the in-plane anisotropy $\mathcal{K}_{4, \operatorname{dip}}(T, B)$, which will be calculated as a function of the temperature $T$ and the external magnetic field $B$.

It is important to mention that the mean field theory as described in the preceeding subsection does not fulfill the Mermin-Wagner-theorem for the isotropic 2D Heisenberg magnet. In this case the long range magnetic order becomes unstable against with long wavelengths $3 \mathrm{~B}^{3}$. Thus, the consideration of 
these spin waves is very important for the magnetic behavior of a ferromagnetic monolayer. For the determination of the dipole-coupling-induced in-plane anisotropy we apply in addition a many-body Green's function theory to the Hamiltonian Eq.(11), after the lattice is rotated by the in-plane angle $\phi$, Eq.(2). We consider the Green's functions $G_{i j}^{\alpha-}=\left\langle\left\langle S_{i}^{\alpha} ; S_{j}^{-}\right\rangle\right\rangle, \alpha=+_{-}, z$, which are solved within the Tyablikov-decoupling11. Since interactions between spin waves are partly taken into account, the magnetic properties can be determined up to the Curie temperature by this method. The respective formalism is described in detail int, thus we outline merely necessary extensions in the Appendix. The magnetization $m(T, B, \phi)$, the internal energy $E(T, B, \phi)$, and the free energy $F(T, B, \phi)$ are calculated. For comparison, we determine the corresponding quantities alse-by considering the Holstein-Primakoff approximation 14, which is valid at low temperatures.

\section{RESULTS AND DISCUSSION}

By application of the described methods we calculate the effective dipole-coupling-induced in-plane magnetic anisotropy $\mathcal{K}_{4, \text { dip }}(T, B)$ for a square ferromagnetic monolayer as a function of the temperature $T$ and the applied magnetic field $B$. A finite value indicates that this anisotropy is caused by the magnetic dipole coupling, since other sources of magnetic anisotropies are not present in the Hamiltonian Eq.(1). For the strength of the dipole interaction we choose $\left(g \mu_{B}\right)^{2} / a_{o}^{3}=w=0.01 J$, which is appropriate for $3 d-$ transition metal ferromagnets. $\mathcal{K}_{4, \operatorname{dip}}(T, B)$ is given in units of the energy difference between the perpendicular and the in-plane magnetization (demagnetizing energy) $E_{\text {demag }}(0)=(3 / 2) w S(0,0) m^{2}(0) \approx 2 \pi w S^{2}$ for a ferromagnetic monolayer at $T=0$.

The free energy $F(T, \phi)$ exhibits a four-fold symmetry as a function of the in-plane angle $\phi$. The easy magnetic axes are directed along the edges $(\phi=0, \pi / 2$, etc. $)$, and the hard axes along the diagonals $(\phi=\pi / 4,3 \pi / 4$, etc.) of the square lattice as obtained ind. The quartic anisotropy $\mathcal{K}_{4, \text { dip }}(T, B)$ is depicted in Fig.1 as a function of the temperature, calculated by the Oguchi approach. Three different sizes of the spin cluster are considered $(N=4,9,16)$, as well as different magnetic field strengths $B$. The Curie temperature $T_{C}(N)$ decreases with an increasing $N$, since additional spin correlations are considered. As can be seen, $\mathcal{K}_{4, \text { dip }}(T, B)$ increases with an increasing temperature, reaching a maximum at $T_{C}$. This temperature behavior is in striking contrast to the corresponding behavior of other anisotropic contributions. Usually the effective anisotropies decrease with an increasing temperature, and vanish at $T_{C}$ for $B=0$. $\mathcal{K}_{4, \operatorname{dip}}(T, B)$ exhibits a cusp at $T_{C}$ and decreases for temperatures $T>T_{C}$. By increasing the size $N$ of the spin cluster, $\mathcal{K}_{4, \text { dip }}(T, B)$ becomes larger. An application of a magnetic field reduces $\mathcal{K}_{4, \operatorname{dip}}(T, B)$, and the cusp near

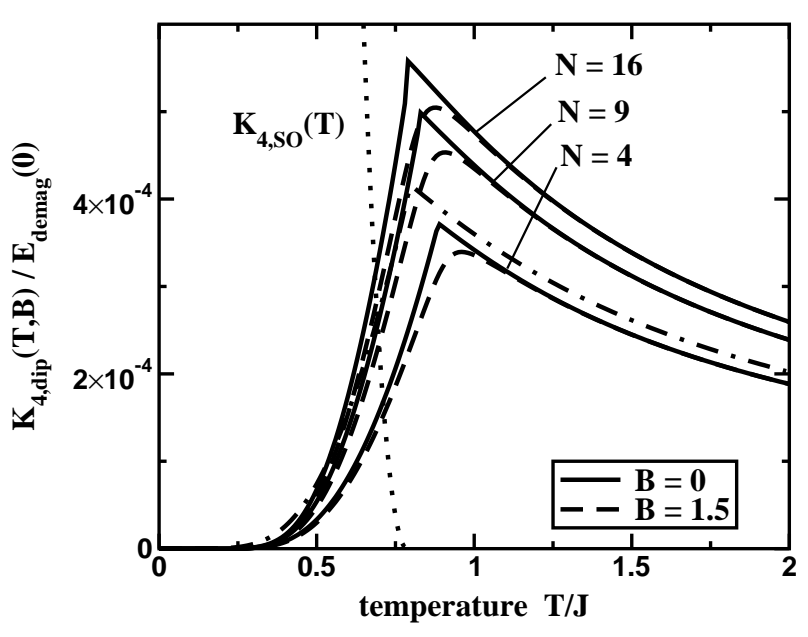

FIG. 1: Effective dipole-coupling-induced quartic in-plane magnetic anisotropy $\mathcal{K}_{4, \text { dip }}(T, B)$ for a square monolayer as a function of the temperature $T$, calculated within the Oguchi mean field method for spin cluster sizes $N=$ 4, 9, 16. Two different magnetic field strengths $B$ are considered $\left(g \mu_{B} B / E_{\text {demag }}(0)=0,1.5\right)$. The field energy and $\mathcal{K}_{4, \text { dip }}(T, B)$ are given in units of the demagnetizing energy $E_{\text {demag }}(0)$. The dot-dashed line shows $\mathcal{K}_{4, \text { dip }}(T, B)$ for $B=0$ and $N=4$ by considering non-diagonal matrix elements of the Hamiltonian, cf. Sec.II. For comparison, also the effective single-ion quartic anisotropy $\mathcal{K}_{4, s o}(T)$ resulting from the spinorbit interaction is shown, calculated within a thermodynamic perturbation theory by assuming $\mathcal{K}_{4, \text { so }}(0)=0.01 E_{\text {demag }}(0)$ (dotted line).

$T_{C}$ changes to a maximum. For comparison, in Fig.1 we show $\mathcal{K}_{4, \operatorname{dip}}(T, B)$ for $B=0$ and $N=4$ by considering also non-diagonal elements in the cluster Hamiltonian $\mathcal{H}^{(4)}$, cf. Sec.II. A decrease of $T_{C}$ and an increase of $\mathcal{K}_{4, \text { dip }}(T, B)$ by $\sim 10 \%$ is obtained with respect to the calculations which consider diagonal elements only.

In addition, we have calculated $\mathcal{K}_{4, \text { dip }}(T, B)$ by application of the many-body Green's function theory as outlined in 4 and in the Appendix. For $w=0.01 \mathrm{~J}$ the Curie temperature is calculated to be $k_{B} T_{C}^{R P A}=$ $0.373 \mathrm{~J}$. In Fig.2(a,b) we present the resulting magnetization $m(T, B)$ and anisotropy $\mathcal{K}_{4, d i p}(T, B)$ as functions of the temperature and the magnetic field. In accordance with the results obtained from the Oguchi approach, $\mathcal{K}_{4, \text { dip }}(T, B)$ increases with an increasing temperature. Due to the consideration of quantum mechanical spins a finite value of $\mathcal{K}_{4, \operatorname{dip}}(T, B)$ is already present at $T=0$, resulting from the zero-point spin motion (quantum fluctuations). Note that the presence of the dipole coupling causes anon-saturated magnetization $m(T=0)<S=1 / 2^{3} \omega^{4}$. In Fig.2 also the results as obtained from the Holstein-Primakoff approximation are shown, which neglects interactions between spin waves. The corresponding magnetization $m(T, B)$ and anisotropy $\mathcal{K}_{4, d i p}(T, B)$ at low temperatures are close to the results as obtained from the RPA. At elevated temperatures $T \gtrsim T_{C} / 3$ the Holstein-Primakoff approxima- 


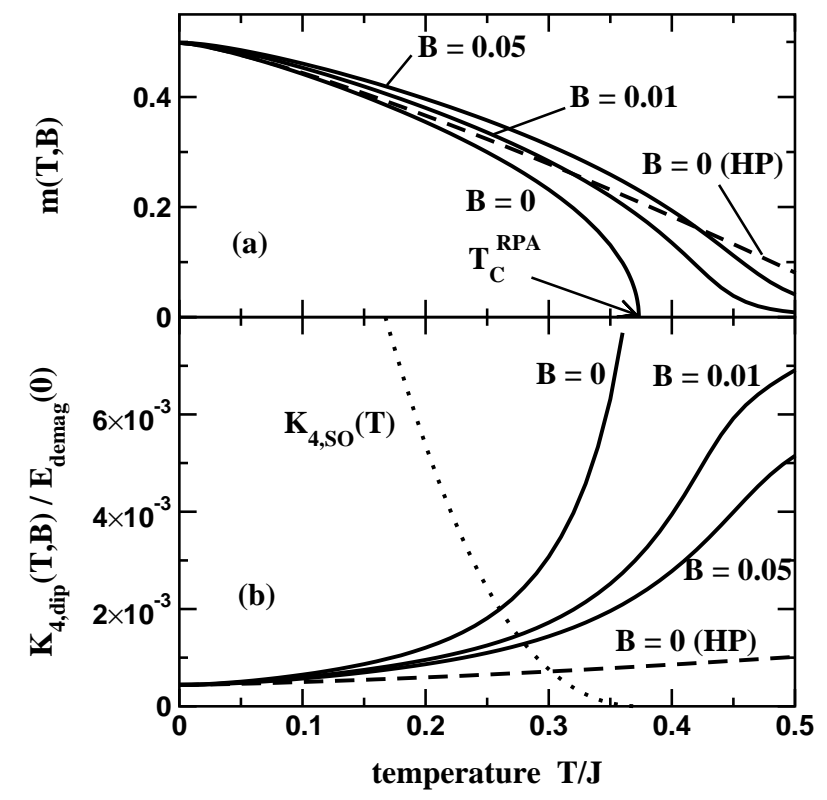

FIG. 2: Magnetization $m(T, B)$ (a) and effective quartic in-plane magnetic anisotropy $\mathcal{K}_{4, d i p}(T, B)$ (b) for a square monolayer as functions of the temperature $T$ and the magnetic field $B$, calculated by application of the Green's function method within the Tyablikov-decoupling (RPA). The Curie temperature is calculated to be $k_{B} T_{C}^{R P A}=0.373 \mathrm{~J}$. The field energy and $\mathcal{K}_{4, \text { dip }}(T, B)$ are given in units of the demagnetizing energy $E_{\text {demag }}(0)$. In addition, the results as obtained from the Holstein-Primakoff approximation (HP) for $B=0$ are depicted (dashed lines). For comparison, the dotted line shows the effective single-ion quartic anisotropy $\mathcal{K}_{4, s o}(T)$, with $\mathcal{K}_{4, \text { so }}(0)=0.01 E_{\text {demag }}(0)$.

tion is no longer valid.

As has already been obtained from Monfe Carlo calculations and interacting spin wave theoryt, the dipolecoupling-induced in-plane anisotropy is caused by magnetic fluctuations. This order-by-disorder effect is thus controlled by the magnetic entropy of the system $\mathrm{B}$. An energetic influence should become apparent at $T=0$ already within a mean field approximation. Besides the effects resulting from the zero-point motion, the terms of the dipole interaction dependent on the in-plane angle $\phi$ cancel for a strictly square magnetic lattice, i.e. for a fully saturated ferromagnetic state. At finite temperatures thermal fluctuations disturb this square periodicity, causing an effective quartic in-plane magnetic anisotropy, which reflects the underlying spatial lattice symmetry. The single-spin mean field theory conserves the periodicity of the square magnetic lattice, since all other spin operators are replaced by uniform expectation values. Thus, within this approximation one obtains $\mathcal{K}_{4, \operatorname{dip}}(T, B)=0$ for all temperatures. On the other hand, within the Oguchi approach the interactions between a number of spins are treated exactly, considering a few collective magnetic excitations with short wavelengths ranging over several lattice constants, and resulting in a finite value for $\mathcal{K}_{4, \text { dip }}(T, B)$ for $T>0$. The many-body Green's function theory takes into account spin waves with all possible wavelengths. Since $\mathcal{K}_{4, \operatorname{dip}}(T, B)$ is maximal at elevated temperatures, $\mathcal{K}_{4, \text { dip }}(T, B)$ is caused by magnetic fluctuations in particular with short wavelengths, which become excited in this temperature range.

Within the Oguchi approach we calculate a maximum value of $\mathcal{K}_{4, \text { dip }}(T, B)$ of the order of $0.1 \%$ of the demagnetizing energy $E_{\text {demag }}(0)$. On the other hand, $\mathcal{K}_{4, d i p}(T, B)$ as calculated from the Green's function method is more than a magnitude larger, namely $\mathcal{K}_{4, \text { dip }}(T, B) \sim$ $0.01 E_{\text {demag }}(0)$ near $T_{C}$. Similarly, the action of a magnetic field $B$ on the anisotropy $\mathcal{K}_{4, \text { dip }}(T, B)$ is calculated to be much more pronounced within the Green's function theory than within the Oguchi approach, cf. Figs.1 and 2. The reason for the strong differences between the results obtained from the two methods is that the former one considers collective magnetic excitations with long wavelengths. These are known to have streng effects on the magnetic properties of ultrathin films 3 . In this case the magnetic field acts merely on spin blocks, i.e. correlated regions of neighboring spins characterized by the short range order parameter 15 , resulting in a much larger magnetic response. For instance, the induced magnetization in a ferromagnetic trilayer has been determined to be an order of magnitude larger by consideration of spin waves 16 . The collective magnetic excitations are most pronounced for a single magnetic layer 3 , the zero-point motion is strongest for the spin number $S=1 / 2$. Note that the theoretical methods applied in the present study are much less demanding than Monte Carlo simulations.

The obtained dipole-coupling-induced in-plane anisotropy is small as compared to other (effective) anisotropies, since we have considered interaction strengths appropriate for $3 d$ - transition metal ferromagnets. A small value for $\mathcal{K}_{4, \operatorname{dip}}(T, B)$ has been conjectured int. If a ten times larger ratio $w / J$ between the dipole coupling strength and the exchange interaction is assumed, the ratio $\mathcal{K}_{4, \text { dip }}(T, B) / E_{\text {demag }}(0)$ increases roughly by the same factor. Since usually the effective anisotropies decrease with an increasing temperature, $\mathcal{K}_{4, \text { dip }}(T, B)$ might become observable at elevated temperatures. For comparison, we show in Figs.1 and 2 also the effective single-ion quartic in-plane anisotropy $\mathcal{K}_{4, s o}(T)$ resulting from the spin-orbit interaction. This quantity is calculated with the help of a thermodynamic perturbation theory 17 . By assuming its strength to be $\mathcal{K}_{4, s o}(0)=0.01 E_{\text {demag }}(0)$, and adapting corresponding Curie temperatures, $\mathcal{K}_{4, s o}(T)$ becomes comparable to $\mathcal{K}_{4, \text { dip }}(T, B)$ at $T / T_{C} \sim 0.8-0.9$. In this temperature range the total quartic in-plane anisotropy should increase again, or exhibit an in-plane magnetic reorientation. As mentioned, this dipole-coupling-induced anisotropy should be more apparent for ferromagnetic (001) thin films with a large $w / J$ - ratio.

We like to comment on the fact that a finite anisotropy is obtained for $T>T_{C}$. Usually the effective anisotropy as observed for a collectively ordered ferromagnetic state vanishes above $T_{C}$. However, a vanishing effec- 
tive anisotropy for $T>T_{C}$ does not indicate that the anisotropy as present in the Heisenberg Hamiltonian disappears. A single (paramagnetic) spin is still subject to e.g. the single-ion uniaxial anisotropy $K_{2}$ also if the net magnetization is zero. The resulting free energy difference between the easy and the hard magnetic directions ('paramagnetic anisotropy') behaves as $\propto K_{2}^{2} / k_{B} T$ for $K_{2} \ll k_{B} T$, and is rather small if $K_{2}$ is small as compared to the exchange interaction $J \propto k_{B} T_{C}$. With regard to the present study, a finite value of $\mathcal{K}_{4, \text { dip }}(T, B=0)$ for $T>T_{C}$ reflects the dipole-coupling-induced anisotropy of a spin block 15 .

Note that the free energy $F(T, B)$ as obtained from the Green's function method yields unphysical results for temperatures near and above $T_{C}$. As can be shown 18 , for large temperatures the free energy $F(T, B)$ as calculated by this method does not approach the value $-k_{B} T \ln 2$, which is the free energy of a single, noninteracting $S=1 / 2$ - spin. This unphysical behavior results in a still increasing $\mathcal{K}_{4, \text { dip }}(T, B)$ for $T>T_{C}$, cf. Fig.2(b). Nevertheless, we expect that the Green's function approach reflects the correct behavior of $\mathcal{K}_{4, \text { dip }}(T, B)$ for $T<T_{C}$, since its behavior is corroborated by the Oguchi method and the Holstein-Primakoff approach.

We have considered a square monolayer only. A similar effect is expected also for thicker films with a square (001) face, or for a hexagonal (111) thin film. In addition, a corresponding effect may emerge for a three-dimensional cubic lattice, for which the dipole interaction cancels exactly. At elevated temperatures, however, caused by thermal fluctuations the frustration due to this periodicity will be lifted. The dipole coupling then induces a cubic magnetic anisotropy with easy axes directed along e.g. the edges of the cubic lattice. Also is this case an increasing anisotropy with an increasing temperature is expected. To obtain an anisotropic contribution caused by the magnetic dipole interaction via the order-by-disorder effect, we emphasize that the lattice symmetry must be strictly cubic or square. A lattice distortion e.g. due to magnetostrictive effects will probably blur this effect.

P.J.J. gratefully acknowledges the invitation and the hospitality of the I. Institute for Theoretical Physics of the Hamburg University. Fruitful discussions with Prof. K.D. Schotte, Free University of Berlin, are acknowledged.

\section{Appendix: Many-body Green's function theory}

The following commutator Green's functions in the frequency space are taken into account: $G_{i j}^{\alpha}(\omega)=$ $\left\langle\left\langle S_{i}^{\alpha} ; S_{j}^{-}\right\rangle\right\rangle_{\omega}, \alpha=+,-, z$. By considering the Hamiltonian Eq.(1), these Green's functions are solved in the usual way by the equation of motion 11,12 . The higher order Green's functions are approximated by the Tyablikov-decoupling (RPA) 11 , e.g. $\left\langle\left\langle S_{k}^{z} S_{i}^{+} ; S_{j}^{-}\right\rangle\right\rangle \simeq$ $\left\langle S^{z}\right\rangle\left\langle\left\langle S_{i}^{+} ; S_{j}^{-}\right\rangle\right\rangle$, resulting in a system of linear equations for the $G_{i j}^{\alpha}(\omega)$. By application of a Fourier transformation into the $2 \mathrm{D}$ wave vector space $\mathbf{k}_{\|} \equiv \mathbf{k}$, one obtains $G^{z}(\mathbf{k}, \omega)=0$, if $\left\langle S^{+}\right\rangle=\left\langle S^{-}\right\rangle=0$. The remaining two Green's functions $G^{ \pm}(\mathbf{k}, \omega)$ are easily evaluated 4 :

$$
\begin{aligned}
G^{+}(\mathbf{k}, \omega) & =2 m(T) \frac{\omega+a(\mathbf{k})}{\omega^{2}-\varepsilon^{2}(\mathbf{k})}, \\
G^{-}(\mathbf{k}, \omega) & =2 m(T) \frac{b(\mathbf{k})}{\omega^{2}-\varepsilon^{2}(\mathbf{k})},
\end{aligned}
$$

with $m(T)=\left\langle S^{z}\right\rangle$. The magnon dispersion relation $\varepsilon(\mathbf{k})$ is given by

$$
\begin{aligned}
& \varepsilon^{2}(\mathbf{k})=a^{2}(\mathbf{k})-b^{2}(\mathbf{k}), \\
& a(\mathbf{k})=g \mu_{B} B+m(T)(J(0)-J(\mathbf{k})) \\
& +w m(T)\left[S(0,0)+S\left(k_{z}, k_{x}\right)\left(1-\frac{3}{2} \cos ^{2} \phi\right)\right. \\
& \left.+S\left(k_{x}, k_{z}\right)\left(1-\frac{3}{2} \sin ^{2} \phi\right)+3 T\left(k_{z}, k_{x}\right) \cos \phi \sin \phi\right], \\
& b(\mathbf{k})=\frac{3}{2} w m(T)\left[S\left(k_{z}, k_{x}\right) \cos ^{2} \phi+S\left(k_{x}, k_{z}\right) \sin ^{2} \phi\right. \\
& \left.-2 T\left(k_{z}, k_{x}\right) \cos \phi \sin \phi\right] \text {. }
\end{aligned}
$$

$J(\mathbf{k})=2 J\left(\cos k_{x} a_{0}+\cos k_{z} a_{0}\right)$ is the Fourier transform of the exchange interaction, with $a_{o}$ the lattice constant. The oscillating lattice sums 13 are defined by

$$
\begin{aligned}
& S\left(k_{x}, k_{z}\right)=\sum_{l, n=-\infty}^{+\infty} \frac{l^{2}}{\left(l^{2}+n^{2}\right)^{5 / 2}} \exp \left(-i k_{x} a_{0} l-i k_{z} a_{0} n\right), \\
& T\left(k_{z}, k_{x}\right)=\sum_{l, n=-\infty}^{+\infty} \frac{l n}{\left(l^{2}+n^{2}\right)^{5 / 2}} \exp \left(-i k_{z} a_{0} l-i k_{x} a_{0} n\right)
\end{aligned}
$$

where the terms with $l=n=0$ have to be omitted. The following expressions are valid for the spin quantum number $S=1 / 2$. The magnetization $m(T)$ is obtained from the spectral theorem 12 :

$$
\begin{aligned}
m(T, \phi) & =\frac{1}{2}-\frac{1}{N} \sum_{\mathbf{k}}\left\langle S^{-} S^{+}\right\rangle(\mathbf{k}) \\
& =\frac{1}{2}-\frac{m(T)}{N} \sum_{\mathbf{k}}\left(\frac{a(\mathbf{k})}{\varepsilon(\mathbf{k})} \operatorname{coth} x-1\right)
\end{aligned}
$$

with $x=\varepsilon(\mathbf{k}) / 2 k_{B} T$. The summation extends over the first Brillouin zone, $N$ denotes the number of k-points. By calculating the expectation value $\left\langle S_{i}^{z} S_{j}^{z}\right\rangle$ with the help of $G^{+}(\mathbf{k}, \omega) 12$, we obtain for the internal energy $E(T, \phi)$ per spin:

$$
\begin{aligned}
& E(T, \phi)=E_{0}+\frac{m(T, \phi)}{2 N} \sum_{\mathbf{k}}\left(e_{1}(\mathbf{k})\left(\frac{a(\mathbf{k})}{\varepsilon(\mathbf{k})} \operatorname{coth} x-1\right)\right. \\
& \left.+\left(e_{2}(\mathbf{k}) \frac{b(\mathbf{k})}{\varepsilon(\mathbf{k})}+\varepsilon(\mathbf{k})\right) \operatorname{coth} x-a(\mathbf{k})+b(\mathbf{k})\right)
\end{aligned}
$$


with the denotations

$$
\begin{aligned}
E_{0}= & -\frac{1}{2} g \mu_{B} B-\frac{1}{8}(J(0)+w S(0,0)), \\
e_{1}(\mathbf{k})= & g \mu_{B} B+\frac{1}{2}(J(0)-J(\mathbf{k})) \\
& +\frac{w}{2}\left(S(0,0)+S\left(k_{z}, k_{x}\right)+S\left(k_{x}, k_{z}\right)\right), \\
e_{2}(\mathbf{k})= & \frac{1}{2} J(\mathbf{k})-\frac{w}{2}\left(S\left(k_{z}, k_{x}\right)+S\left(k_{x}, k_{z}\right)\right) .
\end{aligned}
$$

Finally, the free energy $F(T, \phi)$ is calculated from a temperature integral over $E(T, \phi) 12$. The Holstein-Primakoff approximation 14 is obtained by replacing the magnetization $m(T)$ on the right side of Eq.(13) by its saturation value $S=1 / 2$.
* On leave from the Institut für Theoretische Physik, Freie Universität Berlin, Arnimallee 14, D-14195 Berlin, Germany;

Corresponding author. Electronic adress: jensen@physik.fu-berlin.de

1 See for example, Ultrathin Magnetic Structures $I+I I$, edited by B. Heinrich and J. A. C. Bland (Springer Verlag, Berlin, 1994).

2 C. Herring and C. Kittel, Phys. Rev. 81, 869 (1951); W. Döring, Z. Naturforsch. A16, 1008 (1961).

3 S. V. Maleev, Sov. Phys. JETP 43, 1240 (1976); V. L. Pokrovsky and M. V. Feigel'man, Sov. Phys. JETP 45, 291 (1977); Y. Yafet, J. Kwo, and E. M. Gyorgy, Phys. Rev. B 33, 6519 (1986); P. Bruno, Phys. Rev. B 43, 6015 (1991).

4 Liangbin Hu, Haijin Li, and Ruibao Tao, Phys. Rev. B 60, 10222 (1999).

5 M. Bander and D. L. Mills, Phys. Rev. B 38, 12015 (1988); D. A. Yablonskiy, Phys. Rev. B 44, 4467 (1991); P. Fröbrich, P. J. Jensen and P. J. Kuntz, Eur. Phys. J. B 13, 477 (2000).

6 N. M. Mermin and H. Wagner, Phys. Rev. Lett. 17, 1133 (1966).

7 K. De'Bell, A. B. MacIsaac, I. N. Booth, and J. P. Whitehead, Phys. Rev. B 55, 15108 (1997); A. M. Abu-Labdeh, J. P. Whitehead, K. De'Bell, and A. B. MacIsaac, Phys. Rev. B 65, 024434 (2001); A. Carbognani, E. Rastelli, S. Regina, and A. Tassi, Phys. Rev. B 62, 1015 (2000).

8 J. Villain, Z. Phys. B 33, 31 (1979); C. L. Henley, Phys.
Rev. Lett. 62, 2056 (1989); S. Prakash and C. L. Henley, Phys. Rev. B 42, 6574 (1990).

9 T. Oguchi, Progr. Theor. Phys. 13, 148 (1955).

10 J. S. Smart, Effective field theories of magnetism (Saunders, 1966).

11 S. V. Tyablikov, Ukr. Mat. Zh. 11, 287 (1959); R. TahirKheli and D. ter Haar, Phys. Rev. 127, 88 (1962); H. B. Callen, Phys. Rev. 130, 890 (1963).

12 S. V. Tyablikov, Methods in the quantum theory of magnetism (Plenum Press, New York, 1967); W. Nolting, Quantentheorie des Magnetismus, (B. G. Teubner, Stuttgart, 1986), Vol.2.

13 H. Benson and D. L. Mills, Phys. Rev. 178, 839 (1969); P. J. Jensen, Ann. Physik 6, 317 (1997).

14 T. Holstein and H. Primakoff, Phys. Rev. 58, 1098 (1940).

15 A. M. Polyakov, Phys. Lett. 59B, 79 (1975).

16 P. J. Jensen, K. H. Bennemann, P. Poulopoulos, M. Farle, F. Wilhelm, and K. Baberschke, Phys. Rev. B 60, R14994 (1999).

17 H. B. Callen and E. R. Callen, J. Phys. Chem. Solids 27, 1271 (1966); Y. Millev and M. Fähnle, Phys. Rev. B 51, 2937 (1995); P. J. Jensen and K. H. Bennemann, in Magnetism and Electronic Correlations in Local-Moment Systems: Rare Earth Elements and Compounds, edited by M. Donath, P.A. Dowben, and W. Nolting (World Scientific, Singapore, 1998), pp. $113-140$.

18 P. J. Jensen, unpublished. 